\title{
Spectroscopic Properties of Quercetin-3-O-rhamnoside and Quercetin-3-O-rutinoside in Aerosol-OT Reverse Micelles
}

\author{
Hyoung-Ryun Park, Hai-Bo Liu, ${ }^{\dagger}$ Sung Chul Shin, ${ }^{\dagger}$ Jong Keun Park, ${ }^{\ddagger}$ and Ki-Min Bark ${ }^{\ddagger}, *$ \\ Department of Chemistry and Research Institute of Basic Science, Chonnam National University, Gwangju 500-757, Korea \\ ${ }^{\dagger}$ Department of Chemistry, and Research Institute of Life Science, Gyeongsang National University, Chinju 660-701, Korea \\ ${ }^{\star}$ Department of Chemical Education, and Research Institute of Life Science, Gyeongsang National University, \\ Chinju 660-701, Korea. *E-mail: kiminb@gnu.ac.kr \\ Received December 31, 2010, Accepted January 20, 2011
}

\begin{abstract}
The anomalous spectroscopic properties of quercetin-3-O-rhamnoside (QCRM) and quercetin-3-O-rutinoside (QCRT) in AOT reverse micelle were studied. The excited state intramolecular proton transfer (ESIPT) occurs through the strong hydrogen bond between the $-\mathrm{OH}$ at position 5 and the carbonyl oxygen. Because the ESIPT can only happens in the $S_{1}$ state and the Franck-Condon factor involved in the $S_{2} \rightarrow S_{1}$ internal conversion is small, the $\mathrm{S}_{2} \rightarrow \mathrm{S}_{\mathrm{o}}$ emission alone appears. Because the molecular planarity is improved at the interior of the micelle, the excited state intramolecular charge transfer in the $\mathrm{S}_{1}$ state is extended, and the excited state is more tolerable for any quenching effects in the micelle. Therefore, an $\mathrm{S}_{1} \rightarrow \mathrm{S}_{0}$ emission was newly discovered under this micelle microenvironment. For the $\mathrm{S}_{2} \rightarrow \mathrm{S}_{0}$ emission, the quantum yields increase but the quantum yield of the $\mathrm{S}_{1} \rightarrow \mathrm{S}_{\mathrm{o}}$ emission approximately decreases as the water concentration in the micelle increases.
\end{abstract}

Key Words : Quercetin, AOT reverse micelle, Intramolecular proton transfer, Intramolecular charge transfer

\section{Introduction}

Flavonoids are a group of polyphenols present in substantial amounts $(0.5-1.5 \%)$ in virtually all land-based plants. ${ }^{1,2}$ Besides their important biological roles in plant pigmentation, flavonoids possess anti-cancer, anti-viral and anti-inflammatory properties. ${ }^{3,4}$ Because of their great number and the diversity of their biological activities, these molecules have attracted the great attention of many researchers. Among the large number of various flavonoids, quercetin $\left(3,3^{\prime}, 4^{\prime}, 5,7\right.$-pentahydroxy flavone) is particularly interesting because it is one of the most biologically active and common dietary flavonols. Furthermore, this compound exhibits various anomalous spectroscopic properties. ${ }^{5-7}$ In most cases, flavonoids exist in plants as glycosides, in which one or more hydroxyl groups are joined by a hemiacetal link to a sugar. $^{8}$

Quercetin, and quercetin glycoside, such as quercetin-3$O$-rhamnoside (QCRM, I) and quercetin-3-O-rutinoside (QCRT, II), which contains an -OH group at the position 5 (C-5), have been considered as comprising a special class of nonfluorescent molecules (see Scheme 1). However, as QCRM and QCRT were excited to a 2nd excited state in $\mathrm{CH}_{3} \mathrm{OH}-\mathrm{H}_{2} \mathrm{O}$ and $\mathrm{CH}_{3} \mathrm{CN}-\mathrm{H}_{2} \mathrm{O}$ mixed solvents, a new significant fluorescence emission was discovered. ${ }^{9}$ It is very important to study the physicochemical properties of these molecules in various environments, especially in vivo, so as to provide essential understanding of the mechanism of its strong biological activity. Since it is not easy to do this kind of work in vivo directly, many studies have been done in biologically-mimicking systems such as hydro-organic mixed solvents and various micelles. ${ }^{10-15}$
Quercetin has a -OH group at position 3 (C-3), but QCRM and QCRT have an $O$-glycoside. Besides this difference, quercetin's molecular structure is exactly the same as that of QCRM and QCRT. Any participation of the glucose group in the $\mathrm{S}_{\mathrm{o}} \rightarrow \mathrm{S}_{1}$ or $\mathrm{S}_{\mathrm{o}} \rightarrow \mathrm{S}_{2}$ transition is not observed. ${ }^{8}$ Therefore, glycosylation at C-3 induces only a small hypsochromic shift of this absorption band, in comparison between the absorption spectra and calculated transition wavelengths of these molecules. ${ }^{5,8,9,16-18}$ The theoretical and experimental research papers about the spectroscopic properties of QCRM and QCRT are very small at present time but those outcomes of quercetin are relatively large. Furthermore, the theoretical approach and discussion about the physicochemical properties of quercetin is easy because this molecule has no large substituent such as sugar. Therefore, it is reasonable that the experimental and theoretical results obtained for quercetin can be directly used toward a qualitative explanation of the
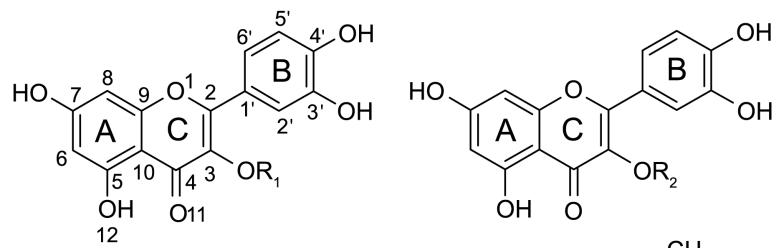

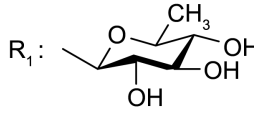

( 1$)$

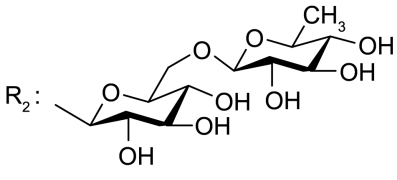

(II)
Scheme 1. The molecular structure of quercetin-3-O-rhamnoside (QCRM, I) and quercetin-3-O-rutinoside (QCRT, II). In quercetin, $\mathrm{R}_{1}$ is $\mathrm{H}$. 
chemical properties of QCRM and QCRT.

Surfactant aggregates in nonpolar solvents assemble to form reverse micelles. Among possible systems, AerosolOT (sodium 1,4-bis[2-ethylhexyl] sulphosuccinate, AOT) in alkanes (e.g. $n$-heptane or isooctane) has been the most widely investigated for its ability to form reverse micellar aggregates in nonpolar solvents, and to solubilize and compartmentalize relatively large amounts of water within their central core. As the molar ratio of $\mathrm{H}_{2} \mathrm{O}$ to AOT $\left(\mathrm{R}=\left[\mathrm{H}_{2} \mathrm{O}\right] /\right.$ [AOT]) increases, the hydrodynamic radius of the spherical aqueous micellar core grows monotonically. ${ }^{19}$ The water solubilized in $\mathrm{AOT} / \mathrm{H}_{2} \mathrm{O} /$ alkane microemulsions is similar in many respects to the interfacial water present near biological membranes or at protein surfaces. ${ }^{20,21}$ The unusual behavior of this water has been attributed to its strong interaction with the ionic head groups of the surfactant and counterions as well as to an overall disruption of the three-dimensional hydrogen-bonded network usually present in bulk water. ${ }^{22-25}$ For this reason, the AOT reverse micelle has been thought to behave as a membrane mimetic system. As the size of the water pool increases, the microviscosity of the solubilized water decreases as its polarity increases. Addition of water also dramatically increases the average aggregation number of the reverse micelles, with little dependence on the hydrocarbon solvent used or the AOT concentration. ${ }^{26}$ Furthermore, this AOT micelle is especially suitable as a solvent system for spectroscopic experiments, because AOT/ $\mathrm{H}_{2} \mathrm{O} /$ alkane ternary systems are homogeneous and optically transparent over a wide range of conditions such as temperature, concentration, etc.

In this work, a comprehensive study of the chemical properties of QCRM and QCRT in the AOT/ $\mathrm{H}_{2} \mathrm{O} /$ heptane reverse micelles with various $R$ values was reported using fluorescence spectroscopy. This study will provide valuable information toward understanding the mechanism underlying the strong biological activities of quercetin glycosides.

\section{Experimental}

QCRM was extracted from houseleeks (Orostachys japonicus A Bergers) by a method described in the literature. ${ }^{27,28}$ The QCRT and NaAOT (SigmaUltra) were purchased from SigmaAldrich (St. Louis, MO, USA) and used without further purification. A few investigations were performed on purified AOT preparations and no difference was observed between these and the unpurified commercially available chemicals. ${ }^{29}$ $n$-Heptane (spectrophotometric grade) and methanol (spectrophotometric grade) were also bought from Sigma-Aldrich and used as received. Deionized, doubly distilled water was used.

The AOT was dried for $48 \mathrm{~h}$ in a vacuum at around $1 \times 10^{-3}$ torr with $\mathrm{P}_{2} \mathrm{O}_{5}$, and kept in a desiccator over $\mathrm{P}_{2} \mathrm{O}_{5}$. A $3 \%(\mathrm{wt} / \mathrm{vol})$ AOT/heptane stock solution was used for all experiments reported in this paper. Low concentration solutions (below $1 \times 10^{-5} \mathrm{M}$ ) were used to avoid any solutesolute interactions and self-absorption. Sample solutions in micelles were prepared by pipetting an appropriate amount of $1 \times 10^{-3} \mathrm{M}$ stock solution into a volumetric flask, evaporating off residual solvent with a gentle stream of $\mathrm{N}_{2}$, and diluting to a specified volume with the $3 \%$ AOT/heptane. A small volume of water was also added to the volumetric flask as needed. Finally, the samples were sonicated for 40 min to ensure that all of the solute was solubilized. In this system, the surfactant/solute ratio is about $1.7 \times 10^{4}$, equivalent to micelle/solute ratio of approximately $740(\mathrm{R}=0)$ and $50(\mathrm{R}=20)$ using the surfactant aggregation number of $23(\mathrm{R}=0)$ and $333(\mathrm{R}=20)$ respectively that was measured for the AOT/alkane solution. ${ }^{30,31}$ This fact ensures that no more than one solute molecule on average occupies a given micelle, precluding significant aggregation effects. Also, under such conditions, solubilization of QCRM and QCRT molecules should cause negligible perturbation of the structure and related properties of the AOT micelles.

Absorption spectra were taken with a JASCO V-530 UV/ visible spectrophotometer with $1.0 \mathrm{~nm}$ spectral bandwidth. Steady-state fluorescence spectra were obtained on a PerkinElmer LS-50B spectrofluorometer. Fluorescence quantum yield was measured using anthracene $(\Phi=0.27)$ as a reference. ${ }^{32-34}$ The fluorescence center of gravity that was proportional to the average energy of emission was calculated as the position of fluorescence emission band..$^{35}$ To measure the fluorescence spectra, the samples were degassed by high purity Ar gas purging for $20 \mathrm{~min}$.

The electronic and geometric structure, and potential energy surface of molecules with excitation were optimized using the single-excitation configuration interaction $(\mathrm{cis}) / 6$ $31 \mathrm{G}^{* *}$ level using Gaussian $03 .{ }^{36}$

\section{Results and Discussion}

The UV-visible absorption spectra in various AOT micelles are shown in Figure 1. The absorption band at $270 \mathrm{~nm}$ is very strong and broad compared with that in hydro-organic mixed solvents. ${ }^{9}$ However, the peak at $365 \mathrm{~nm}$ is so weak that identification and measurement are not easy. The changes in absorption spectra as a function of R value for QCRM and QCRT are similar to each other. As the amount of water in a micelle increases, the absorbance of the $270 \mathrm{~nm}$ band decreases gradually but the shape and band position does not significantly change.

It is well known that fluorescence emission arises from the lowest excited singlet state in complex molecules: this is "Kasha's rule," which has proved to be a very accurate generalization, with few exceptions. ${ }^{37}$ Although QCRM and QCRT had been known as nonfluorescent flavonol, characteristic steady-state fluorescence emission spectra was discovered in AOT micelles when the molecules were excited to the $S_{2}$ state as shown in Figure 2. Similar fluorescence spectra were also found in hydro-organic mixed solvents. ${ }^{9}$ For both molecules, roughly the same broad and structureless emission band is observed around $345 \mathrm{~nm}$. One of the very interesting results of this study is that the new fluorescence emission band around $400 \mathrm{~nm}$ is discovered when the QCRM and QCRT are excited to $S_{1}$ state, as shown in Figure 3. 
(a)

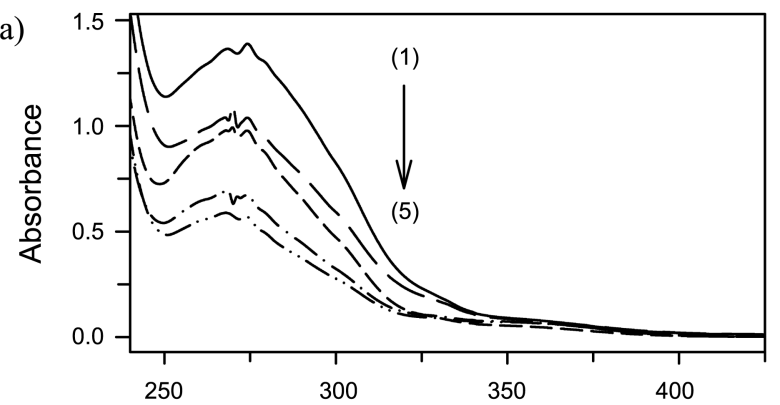

(b)

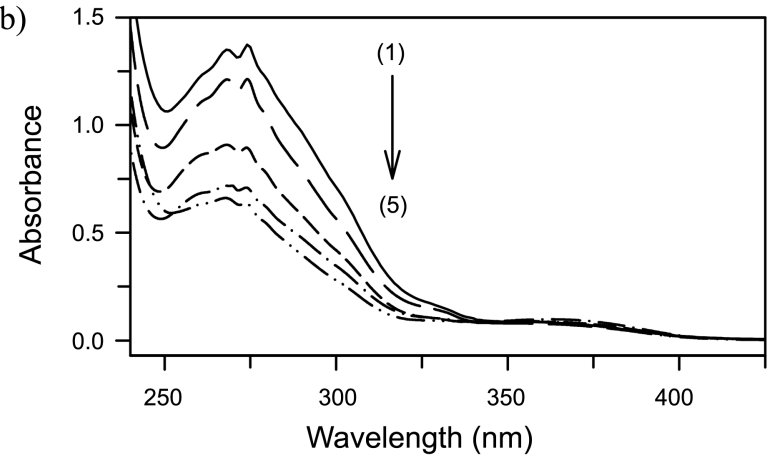

Figure 1. Absorption spectra of QCRM (A), and QCRT (B) in 3\% AOT reverse micelle; (1) $\mathrm{R}=0$ ( $($ ), (2) $\mathrm{R}=4$ ( ---$)$, (3) $\mathrm{R}=7$ ( - - - - ), (4) $\mathrm{R}=15(-\cdot-\cdot),(5) \mathrm{R}=30(-\cdots-\cdots)$.

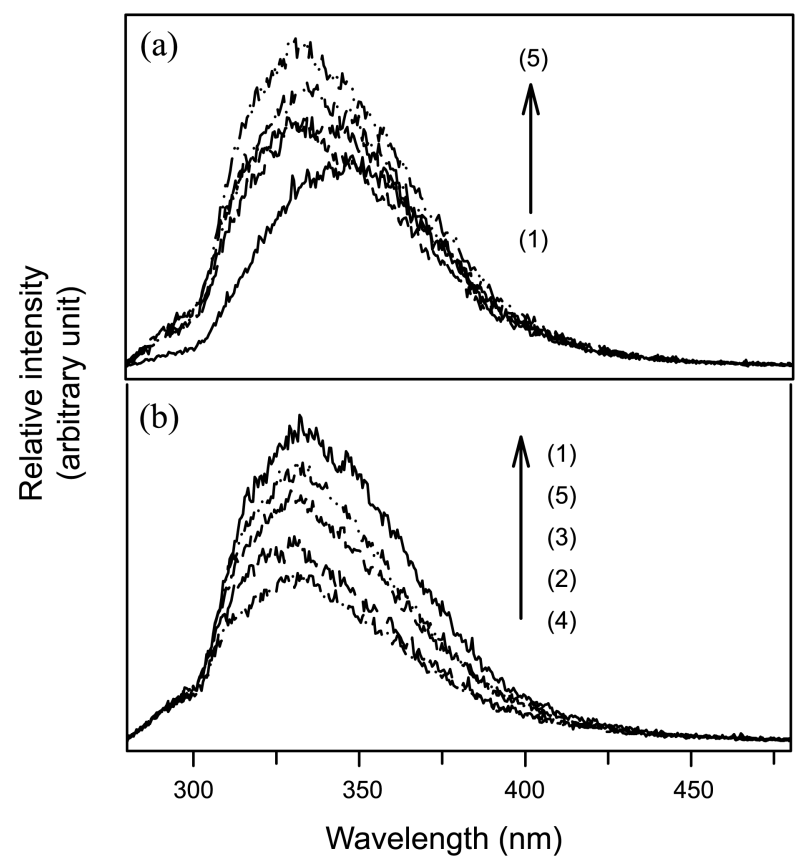

Figure 2. Steady-state fluorescence emission spectra of QCRM (A), and QCRT (B) in 3\% AOT reverse micelle; (1) R=0 ( $\longrightarrow$ ), (2) $\mathrm{R}=4$ ( - - ), (3) $\mathrm{R}=7$ ( - - - ), (4) $\mathrm{R}=15$ (-.- - ), (5) $\mathrm{R}=30(-\cdots-\cdots)$. $\lambda_{\mathrm{ex}}=270 \mathrm{~nm}$.

The intensity of these spectra is about $5-10 \%$ compared with the spectra obtained using $\lambda_{\mathrm{ex}}=270 \mathrm{~nm}$ because the absorbance at $365 \mathrm{~nm}$ is very small. Despite of low intensity, these fluorescence spectra are significant because any obvious background due to Raman scattering or solvent effect is so small that its presence can be ignored. As mentioned pre-

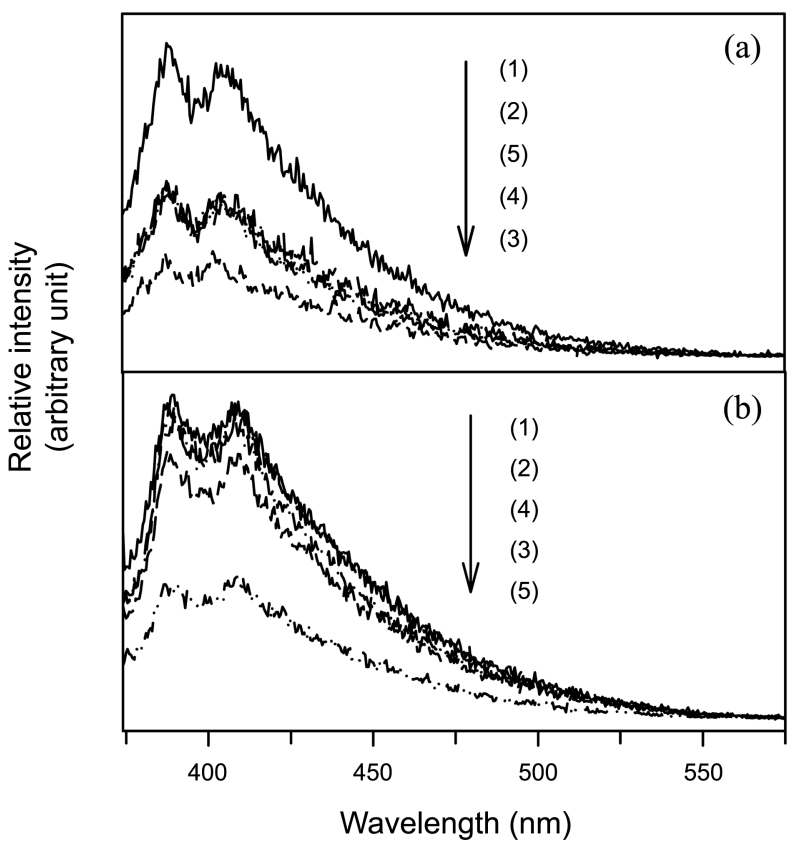

Figure 3. Steady-state fluorescence emission spectra of QCRM (A), and QCRT (B) in 3\% AOT reverse micelle; (1) $\mathrm{R}=0$ ( $\longrightarrow$ ), (2) $\mathrm{R}=4$ (-- -), (3) $\mathrm{R}=7$ ( - - - ), (4) $\mathrm{R}=15$ (-.- - ), (5) $\mathrm{R}=30(-\cdots-\cdots) \cdot \lambda_{\mathrm{ex}}=365 \mathrm{~nm}$.

viously, this kind of fluorescence emission spectra had not been observed in bulk solution. The shape and position of the band are not changed significantly by addition of water in the micelle. Therefore, using two excitation wavelength, 270 and $365 \mathrm{~nm}$, different emission spectra are obtained. When the molecules are excited to the $\mathrm{S}_{2}$ state, the emission corresponding to the $\mathrm{S}_{2} \rightarrow \mathrm{S}_{\mathrm{o}}$ appears only, but if the molecules are excited to the $S_{1}$ state, the $S_{1} \rightarrow S_{0}$ emission band shows clearly, with considerable intensity.

The steady-state spectroscopic results corresponding to $\mathrm{S}_{2} \rightarrow \mathrm{S}_{\mathrm{o}}$ emission $\left(\lambda_{\mathrm{ex}}=270 \mathrm{~nm}\right)$ are shown in Table 1. A small red shift $(\sim 8 \mathrm{~nm})$ of the fluorescence center of gravity is observed for QCRM as the amount of added water in micelle increases. For QCRT, this red shift of the emission band is rather small. Because the shape of fluorescence peaks in spectra are not Gaussian curve, the center of gravity and peak maximum are slightly different. The quantum yields of QCRM and QCRT are similar to each other and larger than those in bulk solution. ${ }^{9}$ Also, for both molecules, the quantum yield increases as more water is added in the micelle. Since the quantum yield is defined by the ratio of the absorbance to the emission intensity, the change of quantum yield is not directly proportional to the strength of fluorescence emission band. The fluorescence quantum yields measured using $365 \mathrm{~nm}$ excitation light are small compared with those in Table 1 but the difference is not large. Due to the very small absorbance at this excitation wavelength, the maximum relative uncertainty is around $30 \%$. So, the data are not shown here. However, as the R value in the micelle increases, the quantum yield decreases approximately, contrary to the quantum yield obtained using $\lambda_{\mathrm{ex}}=270 \mathrm{~nm}$. This result suggests that each fluorescence 
Table 1. The fluorescence quantum yields $(\Phi)$ and fluorescence center of gravity $\left(v_{\mathrm{f}}\right)$ of QCRM and QCRT in AOT reverse micelle. $\lambda_{\text {ex }}=270 \mathrm{~nm}$

\begin{tabular}{rcccc}
\hline \multicolumn{3}{c}{ QCRM } & \multicolumn{2}{c}{ QCRT } \\
\hline $\mathrm{R}^{a}$ & $v_{\mathrm{f}}(\mathrm{nm})^{b}$ & $\Phi\left(10^{-2}\right)^{c}$ & $v_{\mathrm{f}}(\mathrm{nm})^{b}$ & $\Phi\left(10^{-2}\right)^{c}$ \\
\hline 0 & 353 & 1.60 & 350 & 1.43 \\
2 & 350 & 2.12 & 349 & 1.76 \\
4 & 348 & 2.29 & 347 & 1.96 \\
7 & 347 & 2.38 & 345 & 2.36 \\
10 & 346 & 2.60 & 346 & 2.60 \\
15 & 345 & 3.73 & 345 & 3.03 \\
30 & 345 & 4.41 & 346 & 3.35 \\
\hline
\end{tabular}

${ }^{a}$ The concentration ratio of water to AOT. ${ }^{b}$ Uncertainty is $<1 \%$. ${ }^{c}$ Uncertainty is $<4 \%$.

emission measured using $\lambda_{\mathrm{ex}}=270$ and $365 \mathrm{~nm}$ light originates from different excited states.

To understand the spectroscopic properties of QCRM and QCRT, it is very helpful to think about the position that these solute molecules occupy inside of the AOT micelle. Because QCRM and QCRT are insoluble in $n$-heptane but are soluble in water owing to the many polar substituents, these molecules are not found outside the micelles or even in the middle of the alkyl tails of the surfactants. It has been reported that fluoroquinolone antibiotics, which are similar in solubility and molecular size to the quercetin, are intimately associated with the micelles and resides at the micellar interface near the AOT headgroups. ${ }^{25}$ Since quercetin will be located at the similar position as the fluoroquinolones, it is reasonable to assume that the main part of QCRM and QCRT molecule except the $O$-glycoside that strongly interact with the micellar interface do not necessarily reside in a well-defined water pool even though the water concentration is large.

Since intramolecular hydrogen bonding is known to be a more important stability criterion than is steric hindrance, examination of this hydrogen bonding will be one of the most important tasks to understand the characteristic photochemical properties. ${ }^{8,9}$ The determination of the crystal structure of quercetin by X-ray diffraction, and theoretical calculations of its structure in liquid solution, confirmed the formation of a strong intramolecular hydrogen bond between the $-\mathrm{OH}$ group at the $\mathrm{C}-5$ and the carbonyl oxygen $(\mathrm{O}-$ 11) at position 4 (C-4), which allows the formation of a stable six-membered ring. ${ }^{5,9,16,38,39}$ For QCRM and QCRT, it has been verified that besides this intramolecular hydrogen bond, several other intramolecular hydrogen bonds are also observed between the $-\mathrm{OH}$ of the glucose and carbonyl oxygen, or another $-\mathrm{OH}$ of the sugar and the $-\mathrm{OH}$ at the $\mathrm{B}$ ring. Nevertheless, the most stable molecular structure in the ground state is an intramolecularly hydrogen-bonded closed conformer between the $-\mathrm{OH}$ at the $\mathrm{C}-5$ and $\mathrm{O}-11$. Due to this reason, the excited state behavior of QCRM and QCRT is dramatically different from that of 3-hydroxyflavone and related compounds, which have only $-\mathrm{OH}$ group at the $\mathrm{C}-3$, not at the C-5., $\mathrm{It}$ has been known that excited state intra-

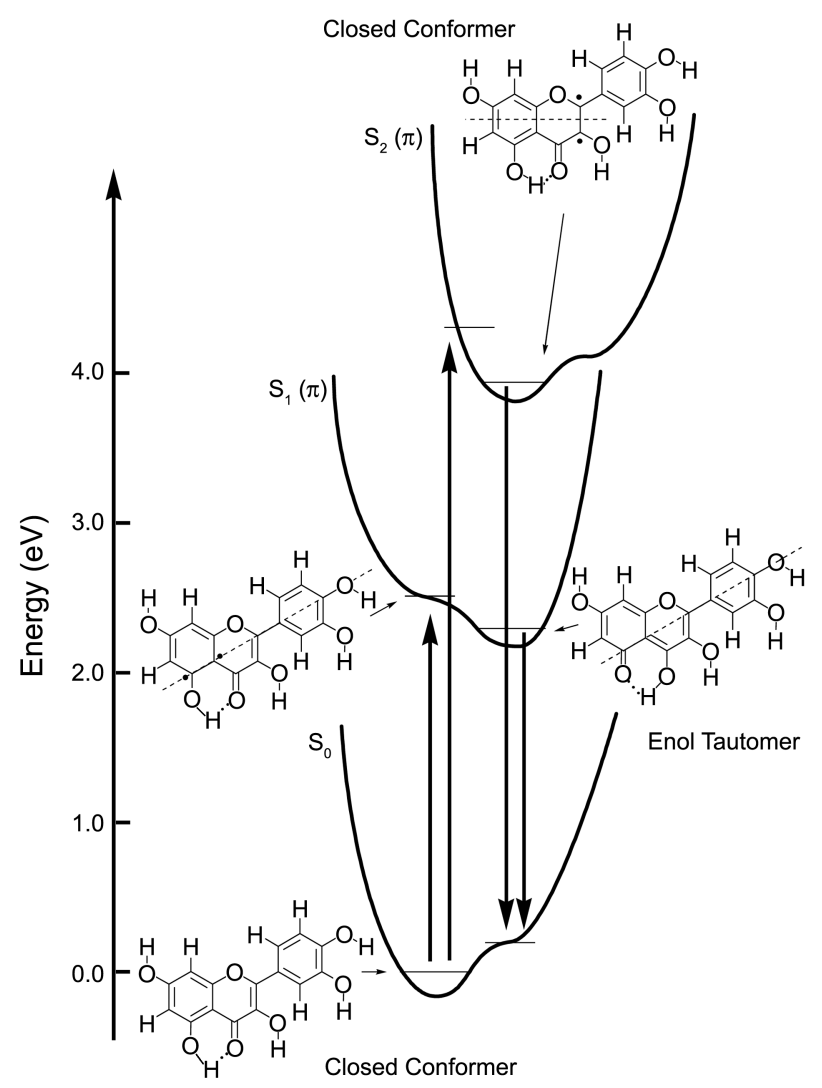

Figure 4. Schematic representation of $\mathrm{S}_{0}, \mathrm{~S}_{1}{ }^{(\pi)}$, and $\mathrm{S}_{2}{ }^{(\pi)}$ potential energy surfaces for quercetin in the gas phase. Dotted line $(----)$ denotes the nodal plane, and $\operatorname{dot}(\bullet)$ indicates the lone electron.

molecular proton transfer (ESIPT) by tautomerization has been observed in many molecules having this kind of intramolecular hydrogen bonding, such as $o$-hydroxybenzaldehyde, salicylic acid, 9-hydroxyphenalenone, polyhydroxyflavone etc. ${ }^{40-50}$ Furthermore, in 5-hydroxyflavone, direct evidence of the ESIPT between this -OH at the C-5 and O11 was observed. ${ }^{40}$ Since QCRM and QCRT are also 5hydroxyflavones, it can be assumed that a similar ESIPT will occur in these molecules.

The intramolecular proton transfer is a "keto-enol tautomerization"-along with the proton transfer, a significant redistribution of electron density results in the changes of the bond orders and length of heavy atoms such as carbon and oxygen. The correct description of electronic structure of such a molecule involves contributions from both of the limiting enol and keto forms. The relative stability of these two forms was predicted using "nodal plane" model. ${ }^{41,43}$ To verify the appearance of ESIPT in QCRM and QCRT, this "nodal plane" model was applied to quercetin. The schematic representation of $\mathrm{S}_{0}, \mathrm{~S}_{1}{ }^{(\pi)}$, and $\mathrm{S}_{2}{ }^{(\pi)}$ potential energy surfaces for quercetin in the gas phase are shown in Figure 4. The energy gap between different electronic states, especially between $\mathrm{S}_{0}$ and $\mathrm{S}_{1}$, is smaller than that of QCRM and QCRT as estimated from absorption and fluorescence spectra. This difference is acceptable, because the glycosylation at C-3 will induce some hypsochromic shift of the $\mathrm{S}_{0} \rightarrow \mathrm{S}_{1}$ absorp- 
tion band, and the environment of the solute molecule will also cause some change in the absorption and emission band position. ${ }^{9}$ The occurrence of ESIPT can be understood by considering the $\pi$ electron nodal pattern of the wavefunction. In this figure, the nodal plane at the $\mathrm{S}_{1}{ }^{(\pi)}$ and $\mathrm{S}_{2}{ }^{(\pi)}$ state is drawn as a dotted line. In the $S_{1}{ }^{(\pi)}$ state, the apparent $\pi$ lone electrons will be localized at $\mathrm{C}_{5}$ and $\mathrm{C}_{10}$ atoms. The lone electrons facilitate the rearrangement of bonds to produce the enol tautomer. This rearrangement and delocalization of the lone electrons significantly lower the energy of the excited state and makes the potential energy surface of the $\mathrm{S}_{1}{ }^{(\pi)}$ state largely distorted from that of the $\mathrm{S}_{\mathrm{o}}$ state. In contrast, the wavefunction in the $\mathrm{S}_{2}{ }^{(\pi)}$ state show that the nodal plane passes the middle between the $\mathrm{C}_{6}$ and $\mathrm{C}_{7}$ atoms, and $\mathrm{C}_{2}$ and $\mathrm{C}_{3}$ atoms. The ESIPT to produce the enol tautomer in the $\mathrm{S}_{2}{ }^{(\pi)}$ state cannot take place, because the formation of double bonds between $\mathrm{C}_{4}$ and $\mathrm{C}_{10}$ atoms, and $\mathrm{C}_{5}$ and $\mathrm{O}_{12}$ atoms is not possible. As a result, the potential energy surface of the $S_{2}{ }^{(\pi)}$ state is similar to that of the $S_{o}$ state. Therefore, this theoretical approach demonstrates that the closed conformer-the keto form-undergoes tautomerization in the $S_{1}$ state because the nodal plane of the wavefunction stabilizes the enol tautomer only in the first excited state $\left(\pi, \pi^{*}\right){ }^{41,43}$ Eventually, the $\mathrm{S}_{2}$ state will be much less susceptible to ESIPT than the $\mathrm{S}_{1}$ state. In the case of QCRM and QCRT, the same ESIPT will occur in the $\mathrm{S}_{1}$ state. The lack of $\mathrm{S}_{1} \rightarrow \mathrm{S}_{\mathrm{o}}$ fluorescence emission of 5-hydroxyflavones just as in QCRM and QCRT had been explained using the formation of a distorted excited state due to the near degeneracy of $\pi, \pi^{*}$ and $n, \pi^{*}$ states. ${ }^{5,9}$ This would confirm that this distorted excited state in the $S_{1}$ state is formed by this ESIPT. Therefore, the anomalous fluorescence properties of 5-hydroxyflavones are mainly caused by this specific excited state dynamic process.

The geometrical structure of QCRM and QCRT, especially the dihedral angle $\theta$, which defines the position of the $B$ ring with regard to the chrome part ( $\mathrm{A}$ and $\mathrm{C}$ rings), is determined through several effects: the repulsion caused by the important steric hindrance between the $-O$-glycoside at the C-3 and B ring, which would lead to a staggered conformation, the various intramolecular hydrogen bonding, and the effect of electronic conjugation which tends to bring the $\mathrm{B}$ ring into the chromone plane. Due to these various opposing effects, the B ring of QCRM and QCRT deviates slightly from the plane of the chrome part in the ground state. However, the B ring will easily become coplanar with the $\gamma$-pyrone ring (A and $C$ rings) because the dihedral angle will be small. This statement is supported by the theoretical calculation. The molecular orbital of quercetin in the vapor phase shows that $\theta$ is $24.8^{\circ}$ in $\mathrm{S}_{0}, 0.0^{\circ}$ in $\mathrm{S}_{1}{ }^{(\pi)}$ and $17.7^{\circ}$ in $\mathrm{S}_{2}{ }^{(\pi)}$. So, in the $\mathrm{S}_{1}$ state, the $\mathrm{B}$ ring is on the same level with the $\gamma$-pyrone ring; in the $S_{2}$ state, this angle is also small. Therefore, the B ring of quercetin in the vapor phase easily becomes approximately coplanar with the main heteroatom ring system for a large part of the time while in the excited state.

The absorption bands of QCRM and QCRT show red shift
(10 $15 \mathrm{~nm})$, and the emission band corresponding to $\mathrm{S}_{2} \rightarrow$ $\mathrm{S}_{\mathrm{o}}$ exhibits a large blue shift $(\sim 20 \mathrm{~nm})$ in the AOT micelle compared with those in the hydro-organic mixed solvents. ${ }^{9}$ So the Stokes' shift of these molecules in micelles is much smaller than those in bulk solutions. Within a dielectric continuum approximation of the solvents, the steady-state Stokes' shift can be related to the dipole moment change of the probe molecule upon excitation according to the LippertMataga equation. ${ }^{11}$ Also, contrary to the spectra in bulk solvent, the shape of the absorption and emission spectra become relatively similar to each other in the AOT micelle. These facts suggest that the change of the geometry and dipole moment due to excitation in the micelle will be smaller than those in the hydro-organic solvents. Although the cavity inside the micelle is sufficiently large, the dihedral angle $\theta$ of the QCRM and QCRT at the micellar interface will decrease, since the rotation of the $\mathrm{C}-\mathrm{C}$ bond linking the B- and C-ring is limited and the planarity of the whole molecule is improved. ${ }^{14,15,25}$ Therefore, the distortion of the aromatic ring due to ESIPT at the $\mathrm{S}_{1}$ state will be somewhat restricted, and the electronic conjugation throughout the molecule further extended.

Since the QCRM and QCRT have both an electron donor and acceptor, and at the micelle, the B ring becomes closely coplanar with the main heteroatom ring system in the excited state, these molecules will be good donor-acceptorconjugated molecules. The initial molecular species $(\mathrm{N})$ in the excited state will be changed to the charge transferred zwitterionic form (T) induced by the excited state intramolecular charge transfer (ESICT), as shown in Scheme 2. ${ }^{9}$ The $\mathrm{T}$ form produced in this way will be stabilized further, owing to the larger delocalization of the $\pi$ electrons. Since the ESIPT seldom occurs at the $\mathrm{S}_{2}$ state, the fluorescence properties at this state will mainly be determined by ESICT. Usually, the additional resonance forms due to ESICT lead to a stronger fluorescence emission because the excited state becomes more stable and more tolerable for any quenching effects. Moreover, the Franck-Condon factors involved in the $S_{2} \rightarrow S_{1}$ radiationless transition will be very small in the AOT micelle for the following reasons: First, the large molecular geometry change between $S_{1}$ and $S_{2}$ state may reduce the vibrational overlap integrals. Second, the energy gap between the zero-point vibrational levels of the $S_{1}$ and $S_{2}$ states will be relatively large owing to the ESIPT in the $S_{1}$ state. Under these circumstances, the $S_{2} \rightarrow S_{1}$ internal conversion of QCRM and QCRT will be very slow. Therefore, strong $\mathrm{S}_{2} \rightarrow \mathrm{S}_{\mathrm{o}}$ fluorescence can be exhibited in the AOT micelle.

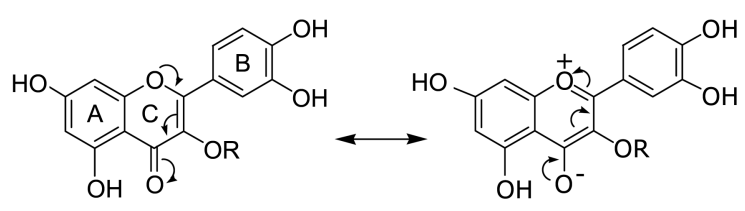

(N)

Scheme 2. The excited state intramolecular charge transfer. 
ESICT will also provide good evidence to explain the appearance of $\mathrm{S}_{1} \rightarrow \mathrm{S}_{\mathrm{o}}$ emission in the AOT micelle. Because the electronic conjugation by ESICT at the $\mathrm{S}_{1}$ and $\mathrm{S}_{2}$ state should become very broad and strong in the micelle, the fluorescence emission and absorption intensity is usually enhanced. ${ }^{14,15,51}$ Therefore, the absorbance of the $270 \mathrm{~nm}$ band and quantum yields for $\mathrm{S}_{2} \rightarrow \mathrm{S}_{\mathrm{o}}$ emission at the AOT micelle are larger than those in bulk solution, as seen in Figure 1 and Table 1. If the twist of the aromatic ring is limited at the interior of the micelle, ESIPT will not occur very strongly. Therefore, since the specific radiationless pathway in the $S_{1}$ state will not be greatly activated, the probability to produce $S_{1} \rightarrow S_{0}$ emission may greatly increase. Furthermore, if the difference in nuclear configuration between the ground and excited states decreases in the AOT micelle, the rate of radiationless deactivation processes will be relatively slow. ${ }^{5,9}$ Also, at the interior of the AOT micelle, any quenching effect by the solvent and other molecules present in the system decreases since the amount of collisions between the solute and any other molecules will decrease. Owing to all of these reasons, the $\mathrm{S}_{1} \rightarrow \mathrm{S}_{\mathrm{o}}$ fluorescence emission can be observed in the AOT micelle.

Because of the absence of ESIPT at the $\mathrm{S}_{2}$ state, the ESICT will have a major influence on the change of quantum yields for the $S_{2} \rightarrow S_{0}$ emission as a function of water concentration in the micelle. To understand this excited state decay process, think about the variation in micellar structure according to the $\mathrm{R}$ values. When $\mathrm{R}$ is small, the packing of the interfacial region at the AOT reverse micelles is rather tight. It has been known that the hydrocarbon tails of the AOT aggregates at small $\mathrm{R}$ values can be wide open with enhanced segmental mobility allowing solvent penetration. ${ }^{52}$ This allows the solute molecules embedded in this region to approach the polar head groups in the micelle. In this case, the intermolecular hydrogen bond and dipole-dipole interaction between the polar groups of solutes, the $\mathrm{H}_{2} \mathrm{O}$, counter cations and polar head groups of the surfactant molecules will be strong. These kinds of intermolecular interactions will diminish the intramolecular hydrogen bond between the $-\mathrm{OH}$ at $\mathrm{C}-5$ and $\mathrm{O}-11$ at the $\mathrm{C}-4$. As a result, the dihedral angle, $\theta$, will be relatively large, and the ESICT will be limited to some extent. Finally, the radiative decay rate becomes slow, and the quantum yields are small at the small $R$ values. Because increasing the amount of water in the micelle results in the decrease of segmental mobility of the hydrocarbon tail of surfactant molecule, the phase continuous with the solute molecules is pushed or squeezed out into the nonpolar phase. ${ }^{52}$ In relatively nonpolar environments, the external hydrogen bonding perturbation described above will be reduced, and the number of molecules having various strong intramolecular hydrogen bonding will increase. Since the main part of QCRM and QCRT (A, C and B ring) is squeezed out into the nonpolar phase due to the increase of water concentration in micelles, the B ring will more easily become coplanar with the $\gamma$-pyrone ring. Since the ESICT at the $S_{2}$ state should occur more easily and satisfactorily in this case, the quantum yields for $\mathrm{S}_{2} \rightarrow \mathrm{S}_{0}$ emission increase gradually as added water in the micelle increases.

For the change of fluorescence properties of QCRM and QCRT in the $\mathrm{S}_{1}$ state, the ESIPT will have stronger effect than the ESICT in the micelle because the ESIPT occurs only in the $\mathrm{S}_{1}$ state. The ESIPT in the $\mathrm{S}_{1}$ state happens more easily and effectively as the water content in AOT micelle increases because the molecules having strong intramolecular hydrogen bond between the $-\mathrm{OH}$ at the $\mathrm{C}-5$ and $\mathrm{O}-11$ will increase. Since the effective nonradiative decay from $S_{1}$ state has been explained using the distortion of molecular skeleton during the ESIPT, the quantum yield for $\mathrm{S}_{1} \rightarrow \mathrm{S}_{\mathrm{o}}$ emission decreases roughly due to the increase of added water in the micelle when both QCRM and QCRT are excited to the $S_{1}$ state (The data are not shown).

\section{Conclusions}

In the AOT reverse micelle, although the only $\mathrm{S}_{2} \rightarrow \mathrm{S}_{\mathrm{o}}$ emission is observed when the molecules are excited to the $\mathrm{S}_{2}$ state, $\mathrm{S}_{1} \rightarrow \mathrm{S}_{0}$ emission spectra is also exhibited as the molecules are pumped to the $S_{1}$ state. These anomalous fluorescence properties of QCRM and QCRT are caused by the specific photochemical and photophysical properties such as ESIPT and ESICT. Using the "nodal plane" model, the ESIPT for quercetin can be described theoretically as a "keto-enol tautomerization". This theoretical approach shows that the $\mathrm{S}_{1}$ state is more susceptible to ESIPT compared with the $S_{2}$ state, because the nodal plane of the wavefunction stabilizes the enol tautomer only in the $S_{1}$ state. Since the structure of the aromatic ring is distorted due to ESIPT in the $\mathrm{S}_{1}$ state, $\mathrm{S}_{1} \rightarrow \mathrm{S}_{\mathrm{o}}$ fluorescence of QCRM and QCRT had not been observed. Also, the Franck-Condon factors involved in the $S_{2} \rightarrow S_{1}$ internal conversion will be very small. Owing to these reasons, QCRM and QCRT can exhibit strong $\mathrm{S}_{2} \rightarrow \mathrm{S}_{\mathrm{o}}$ fluorescence emission in the AOT micelle. The dihedral angle $\theta$ in the excited state is smaller than that in the ground state. Furthermore, since the rotation of the bond between $\mathrm{C}$ 2 and C-1' is limited, and the planarity of the whole molecules is improved at the micellar interface, electronic conjugation such as ESICT will be further extended in the AOT micelle. Due to this specific microenvironment in the interior of the micelle, QCRM and QCRT exhibited significant $\mathrm{S}_{1} \rightarrow$ $\mathrm{S}_{\mathrm{o}}$ emission.

Since the external hydrogen bonding perturbation will decrease due to the increase of water concentration in micelles, the B ring will more easily become coplanar with the $\gamma$-pyrone ring, and the ESICT will be very active as the amount of water in micelle is large. Therefore, when the molecules are excited to $S_{2}$ state, the quantum yields for $S_{2}$ $\rightarrow \mathrm{S}_{\mathrm{o}}$ emission increase as a function of $\mathrm{R}$ value in micelle. Also, when the water concentration in the micelle increases, the ESIPT in the $\mathrm{S}_{1}$ state occurs more easily and the quantum yields for $\mathrm{S}_{1} \rightarrow \mathrm{S}_{\mathrm{o}}$ fluorescence decrease. Because the AOT reverse micelle is regarded as a membrane mimetic system, these kinds of studies for QCRM and QCRT provide much valuable information that should help in understanding the mechanisms of various biological activities. 


\section{References}

1. Thompson, R. S.; Jacques, D.; Haslam, E.; Tanner, R. J. N. J. Chem. Soc. Perkin Trans. 1972, 1, 1387.

2. Jovanovic, S. V.; Steenken, S; Tosic, M.; Marjanovic, B.; Simic, M. G. J. Am. Chem. Soc. 1994, 116, 4846.

3. Middleton, E., Jr.; Kandaswami, C. The Impact of Plant Flavonoids on Mammalian Biology: Implications for Immunity, Inflammation and Cancer; Harborne, J. B., Ed.; Chapman and Hall: London, UK, 1986; p 619.

4. Zare, H. R.; Namazian, M.; Nasirizadeh, N. J. Electroanal. Chem. 2005, $584,77$.

5. Falkovskaia, E.; Sengupta, P. K.; Kasha, M. Chem. Phys. Lett. 1998, 297, 109 .

6. Sengupta, P. K.; Kasha, M. Chem. Phys. Lett. 1979, 68, 382.

7. Smith, G. J.; Markham, K. R. J. Photochem. Photobiol. A 1998, $118,99$.

8. Cornard, J. P.; Boudet, A. C.; Merlin, J. C. J. Mol. Struct. 1999, 508, 37 .

9. Liu, H. B.; Daun, Y.; Shin, S. C.; Park, H. R.; Park, J. K.; Bark, K. M. Photochem. Photobiol. 2009, 85, 934.

10. Bilski, P. L.; Martinez, J.; Koker, E. B.; Chignell, C. F. Photochem. Photobiol. 1998, 68, 20.

11. Park, H. R.; Oh, C. H.; Lee, H. C.; Lee, J. K.; Yang, K.; Bark, K. M. Photochem. Photobiol. 2002, 75, 237.

12. Liu, W.; Guo, R. Colloids Surf. A 2006, 274, 192.

13. Guo, R.; Wei, P.; Liu, W. J. Pharmaceu. Biomed. Anal. 2007, 43, 1580 .

14. Liu, W.; Guo, R. J. Coll. Inter. Sci. 2005, 290, 564.

15. Liu, W.; Guo, R. J. Coll. Inter. Sci. 2006, 302, 625.

16. Cornard, J. P.; Merlin, J. C.; Boudet, A. C.; Vrielynck, L. Biospectros. 1997, 3, 183 .

17. Russo, N.; Toscano, M.; Uccella, N. J. Agric. Food. Chem. 2000 , 48,3232

18. Baranac, J. M.; Petranovi, N. A.; Jasmina, M. M. D. J. Agric. Food Chem. 1997, 45, 1694.

19. Fletcher, P. D. I.; Howe, A. M.; Robinson, B. H. J. Chem. Soc. Faraday Trans. I 1987, 83, 985.

20. Grigolini, P.; Maestro, M. Chem. Phys. Lett. 1986, 127, 248.

21. D’Aprano, A.; Lizzio, A.; Turco Liveri, V.; Aliotta, F.; Vasi, C.; Migliardo, P. J. Phys. Chem. 1988, 92, 4436.

22. Jain, T. K.; Varshney, M.; Maitra, A. J. Phys. Chem. 1989, 93, 7409.

23. Riter, R. E.; Undiks, E. P.; Levinger, N. E. J. Am. Chem. Soc. 1998, 120,6062 .

24. Riter, R. E.; Willard, D. M.; Levinger, N. E. J. Phys. Chem. 1998, $102,2705$.

25. Park, H. R.; Lee, H. C.; Kim, T. H.; Lee, J. K.; Yang, K.; Bark, K. M. Photochem. Photobiol. 2000, 71, 281.

26. Fendler, J. H. Membrane Mimetic Chemistry: Characterization and Applications of Micelles. Microemulsions, Monolayers, Bilayers, Vesicles, Host-Guest Systems, and Polyions, Chapter 3; John Wiley \& Sons: New York, USA, 1982; p 652.

27. Park, H. J.; Young, H. S.; Park, K. Y.; Rhee, S. H. Arch. Pharm. Res. 1991, 14, 167.

28. Jang, S. H.; Lee, S. G.; Kang, J. H.; Park, J. C.; Shin, S. C. Korean J. Med. Crop Sci. 2006, 14, 311.

29. Politi, M. J.; Brandt, O.; Fendler, J. H. J. Phys. Chem. 1985, 89, 2345.

30. Wong, M.; Thomas, J. K.; Nowak, T. J. Am. Chem. Soc. 1977, 99 , 4730.

31. Paulo, P. M. R.; Laia, C. A. T.; Costa, S. M. B. J. Phys. Chem. B 2003, 107, 1097.

32. Bark, K. M.; Forcé, R. K. Spectrochim. Acta 1993, 49A, 1605.

33. Eaton, D. F. Reference Compounds for Fluorescence Measurement; IUPAC Organic Chem. Division: Wilmington, USA, 1987; $\mathrm{p} 1$.

34. Demas, J. N.; Grosby, G. A. J. Phys. Chem. 1971, 75, 2463.

35. Zhang, J.; Bright, F. V. J. Phys. Chem. 1991, 95, 7900.

36. Frish, M. J.; Trucks, G. W.; Head-Gordon, M. H.; Gill, P. M. W.; Wong, M. W.; Foresman, J. B.; Johnson, B. G.; Schlegel, H. B.; Robb, M. A.; Replogle, E. S.; Gomperts, R.; Andres, J. L.; Raghavachari, K.; Binkley, J. S.; Gonzalez, C. R. L.; Fox, D. J.; Defrees, D. J.; Baker, J.; Stewart, J. J. P.; Pople, J. A. Gaussian 03; Gaussian Inc: Pittsburgh, USA, 2003.

37. Bark, K. M.; Forcé, R. K. J. Phys. Chem. 1989, 93, 7985.

38. Rossi, M.; Rickles, L. F.; Halpin, W. A. Bioorg. Chem. 1986, 14, 55.

39. Park, H. R.; Chung, K. Y.; Lee, H. C.; Lee, J. K.; Bark, K. M. Bull. Korean Chem. Soc. 2000, 21, 849.

40. Martinez, M. L.; Studer, S. L.; Chou, P. T. J. Am. Chem. Soc. 1991, 113, 5881.

41. Bisht, P. B.; Petek, H.; Yoshihara, K.; Nagashima, U. J. Chem Phys. 1995, 103, 5290.

42. Nagaoka, S. I.; Nagashima, U. Chem. Phys. 1989, 136, 153.

43. Nagaoka, S. I.; Shinde, Y.; Mukai, K.; Nagashima, U. J. Phys. Chem. A 1997, 101, 3061.

44. Sobolewski, A. L.; Domcke, W. Chem Phys. 1994, 184, 115.

45. Herek, J. L.; Pedersen, S.; Bañares, L.; Zewail, A. H. J. Chem. Phys. 1992, 97, 9046.

46. Barbara, P. F.; Walsh, P. K.; Brus, L. E. J. Phys. Chem. 1989, 93, 29.

47. Catalán, J.; Díaz, C. J. Phys. Chem. A 1998, 102, 323.

48. Vener, M. V.; Scheiner, S. J. Phys. Chem. 1995, 99, 642.

49. Zhu, A.; Wang, B.; White, J. O.; Drickamer, H. G. J. Phys. Chem. $B$ 2004, 108, 895 .

50. Chou, P. T.; Martinez, M. L.; Cooper, W. C. J. Am. Chem. Soc. 1992, 114, 4943.

51. Liu, W.; Guo, R. J. Agric. Food Chem. 2005, 53, 2890.

52. Sarkar, M.; Ray, J. G.; Sengupta, P. K. Spectrochim. Acta part A 1996, 52, 275. 